\title{
Effectiveness of inactivated hantavirus vaccine on the disease severity of hemorrhagic fever with renal syndrome
}

\author{
Yongjin $\mathrm{Yi}^{1}$, Hayne Park ${ }^{1}$, Jaehun Jung ${ }^{2}$ \\ 'Division of Nephrology, Department of Internal Medicine, Armed Forces Capital Hospital, Seongnam, Korea \\ ${ }^{2}$ Department of Preventive Medicine, Armed Forces Medical Command, Seongnam, Korea
}

\begin{abstract}
Background: An inactivated Hantaan virus vaccine (iHV) has been broadly used as a preventive strategy for hemorrhagic fever with renal syndrome (HFRS) by the South Korean Army. After the vaccination program was initiated, the overall incidence of HFRS cases was reduced in the military population. While there are about 400 HFRS cases annually, few studies have demonstrated the efficacy of the iHV in field settings. Therefore, this study aimed to evaluate the iHV efficacy on HFRS severity.

Methods: From 2009 to 2017, HFRS cases were collected in South Korean Army hospitals along with patients' vaccination history. HFRS patients were classified retrospectively into two groups according to vaccination records: no history of iHV vaccination and valid vaccination. Vaccine efficacy on the severity of acute kidney injury (AKI) stage and dialysis events were investigated.

Results: The effects of the iHV on renal injury severity in between 18 valid vaccinated and 110 non-vaccinated patients were respectively evaluated. In the valid vaccination group, six of the 18 HFRS patients (33.3\%) had stage $3 \mathrm{AKI}$, compared to 60 of the 110 (54.5\%) patients in the non-vaccination group. The iHV efficacy against disease progression $\left(\mathrm{VE}_{\mathrm{p}}\right.$ ) was $58.1 \%$ (95\% confidence interval, $31.3 \%$ to $88.0 \%$ ).

Conclusion: The iHV efficacy against the progression of HFRS failed to demonstrate statistically significant protection. However, different severity profiles were observed between the iHV and non-vaccination groups. Additional studies with larger populations are needed to demonstrate the effectiveness of the iHV in patients with HFRS.
\end{abstract}

Keywords: Acute kidney injury, Disease progression, Hemorrhagic fever with renal syndrome, Preventive medicine, Viral vaccine

\footnotetext{
Received May 11, 2018; Revised June 27, 2018;

Accepted August 1, 2018

Correspondence: Yongjin Yi

Division of Nephrology, Department of Internal Medicine, Armed Forces Capital Hospital, 81 Saemaeul-ro, 177 beon-gil, Bundang-gu, Seongnam 13574, Korea.E-mail: uniqueness@gmail.com

ORCID: https://orcid.org/0000-0001-8553-7189

Copyright ( $\odot 2018$ by The Korean Society of Nephrology

(a) This is an open-access article distributed under the terms of the Creative Commons Attribution Non-Commercial License (http://creativecommons.org/licenses/bync-nd/4.0/), which permits unrestricted non-commercial use, distribution, and reproduction in any medium, provided the original work is properly cited.
}

\section{Introduction}

Hantavirus infection causes well-known clinical syndromes including hemorrhagic fever with renal syndrome (HFRS) and hantavirus cardiopulmonary syndrome (HCPS). During the Korean War, thousands of hemorrhagic fever outbreaks were reported in the early 1950s [1]. In 1976, the etiologic agent, Hantaan virus (HTNV), was isolated in lung tissues from the striped field mouse, Apodemus agrarius, by specific immunofluorescent reactions with HFRS patient serum [2]. In the 1980s, more hantavirus species were isolated worldwide from HFRS 
and HCPS outbreaks. Hantaviruses produce a chronic infection in rodent hosts. Rodent reservoirs then transmit hantavirus to humans via aerosolized rodent excreta [3]. HFRS is a clinical syndrome associated with significant acute renal impairment and mortality. Approximately one-third of HFRS cases include stage 3 acute kidney injury (AKI) according to Kidney Disease: Improving Global Outcomes (KDIGO), with $5 \%$ to $10 \%$ overall mortality [4].

Globally, most recent HFRS cases have been reported in East Asia, followed by Russia and Europe. Infections by HTNV, Seoul, Dobrava, and Pluumala viruses have been reported in these areas. In South Korea, HTNV has been the most common causative pathogen of HFRS [5]. Hantavirus is a zoonotic infection hosted by small mammals such as rodents. Humans are generally considered dead-end hosts of hantaviruses as no human-to-human transmission of hantavirus has been reported except for a few reports of HCPS with the Andes virus [6]. Therefore, severity of renal complications and mortality of HFRS patients have been emphasized rather than transmission between humans.

Because no specific therapy has been developed, several prevention strategies have been established including avoiding rodent habitats and inhalation of contaminated dust and vaccine-based immunization. In 1988, the first inactivated HTNV vaccine (iHV), Hantavax ${ }^{\circledR}$ (Korea Green Cross, Seoul, Korea), was developed from cultured brain cells of suckling mice infected with HTNV [7]. Highrisk individuals who were unable to avoid rodent infested areas were provided with iHV in the Republic of Korea (ROK), particularly members of the ROK Army and rural communities [8]. The ROK Army provided additional preventive measures including avoiding training sites in suspected areas of increased HFRS risk, eliminating rodents, and cutting down bushes or tall weeds in adjacent areas [9]. Following the implementation of these efforts, the number of HFRS cases in the ROK Army has declined since the 2000s; however, dozens of HFRS cases still occur annually among the ROK Army population [10]. Furthermore, clinical studies to prove the protective efficacy of the iHV remain inconclusive.

The present study aimed to assess iHV efficacy against disease progression in severe HFRS cases in the ROK Army. To address iHV efficacy, a retrospective study was designed utilizing pooled surveillance data of HFRS cases and comprehensive clinical records from ROK Army hospitals.

\section{Methods}

Vaccine

Each dose of the iHV $\left(\right.$ Hantavax $\left.^{\circledR}\right)$ contains 4,096 enzyme-linked immunosorbent assay (ELISA) units of the formalin-inactivated ROK $84 / 105$ strain. The virus is isolated by direct inoculation onto Vero E6 cells. The virus is then inoculated into 1-day-old suckling mice brains of the Institute of Cancer Research (ICR) strain. After purification by ultrafiltration and sucrose gradient ultracentrifugation, the virus is inactivated with $0.05 \%$ formalin at $4^{\circ} \mathrm{C}$ for fifteen days. Each person receives a $0.5-\mathrm{mL}(8,192$ ELISA units $/ \mathrm{mL}$ ) dose of $\mathrm{iHV}$ via intramuscular injection at 0,1 , and 13 months [7].

\section{Study design and case definition}

This was a population-based, retrospective, cohort study. HFRS cases were enrolled between January 2009 and March 2017. The total size of the ROK army population was approximately 600,000 subjects. More than twothirds of the recruited population were male soldiers in their early twenties (Table 1).

Reporting of HFRS cases for ROK Army personal to the ROK Army Medical Command is mandatory. Inadequate cases were excluded from the study based on the following criteria: 1) cases that did not contain complete record of the patient's iHV vaccination history and medical records at time of admission and 2) cases misdiagnosed serologically due to prior vaccination (classified as false antibody-positive cases). HFRS cases were identified based on the following disease criteria: 1) confirmation of hantavirus infection by polymerase chain reaction test of a patient blood sample or hantavirus-specific antibody tests in a serum sample, and 2) clinical evidence of renal involvement by hantavirus infection (e.g., proteinuria, hematuria, or elevated serum creatinine level).

HFRS-positive subjects were classified into three groups: non-vaccination, valid-vaccination, or invalidvaccination. Patients in the non-vaccination group had no history of iHV vaccination. Patients who had received at least one dose of iHV were classified as vaccinated. According to prior studies on antibody response to the $\mathrm{iHV}$, the vaccination group was divided into valid or invalid groups based on the time between symptom onset 
Table 1. Baseline characteristics of hemorrhagic fever with renal syndrome patients at admission

\begin{tabular}{|c|c|c|c|c|}
\hline Characteristic & $\begin{array}{l}\text { Non-vaccination } \\
\text { group }(\mathrm{n}=110)\end{array}$ & $\begin{array}{l}\text { Valid vaccination } \\
\text { group }(n=18)\end{array}$ & $P$ value & $\begin{array}{l}\text { Invalid vaccination } \\
\text { group }(n=5)\end{array}$ \\
\hline Age $(y r)$ & $21.3(20-22)$ & $23.3(20-23)$ & 0.217 & $21.8(21-21)$ \\
\hline Sex, male & $110(100)$ & $18(100)$ & NA & $5(100)$ \\
\hline Baseline body weight (kg) & $68.9(62.0-74.8)$ & $67.6(62.3-71.5)$ & 0.528 & $67.5(59.0-73.0)$ \\
\hline Body mass index $\left(\mathrm{kg} / \mathrm{m}^{2}\right)$ & $22.5(20.1-24.6)$ & $22.1(19.9-23.4)$ & 0.606 & $22.1(19.3-22.8)$ \\
\hline Rank at admission & & & 0.024 & \\
\hline Private & $25(22.7)$ & $2(11.1)$ & & 0 \\
\hline Private first class & $40(36.4)$ & $4(22.2)$ & & 0 \\
\hline Corporal & $19(17.3)$ & $10(55.6)$ & & $2(40.0)$ \\
\hline Sergeant & $7(6.4)$ & 0 & & $3(60.0)$ \\
\hline Officers & $19(17.3)$ & $2(11.1)$ & & 0 \\
\hline Year at admission & & & 0.539 & \\
\hline $2009-2010$ & $22(20.0)$ & 2 (11.1) & & $3(60.0)$ \\
\hline 2011-2012 & $20(18.2)$ & $4(22.2)$ & & 0 \\
\hline 2013-2014 & $22(29.1)$ & $8(44.4)$ & & $1(20.0)$ \\
\hline $2015-2017$ & $36(32.7)$ & $4(22.2)$ & & $1(20.0)$ \\
\hline
\end{tabular}

Data are presented as mean (interquartile range) or number (\%).

NA, not available.

and last vaccination $[7,11]$. Valid-vaccination group were defined as those who had received the iHV within one year of symptom onset. In contrast, invalid-vaccinated patients were defined as those who had received their last iHV more than one year from time of symptom onset. All vaccination histories were obtained either from vaccination records at the battalion headquarter or from epidemiological investigation records within the command medical office.

Severe cases were defined according to the 2012 KDIGO acute kidney injury (KDIGO-AKI) stages, history of dialysis, and mortality. The group with progressive HFRS cases was defined as 1) patients with KDIGO-AKI stage 3, and 2) patients with AKI who received renal replacement therapies, e.g., conventional hemodialysis or continuous renal replacement therapy.

This study was approved by the institutional review board of the Armed Forces Medical Command (AFMC17082-IRB-17-077). The requirement for informed consent was waived by the review board due to the retrospective study design.

\section{Statistical analysis}

Data were expressed as means and ranges between the 25th and 75th percentiles (interquartile ranges) for con- tinuous variables and as percentages for nominal data. The vaccine effectiveness against disease progression $\left(\mathrm{VE}_{\mathrm{p}}\right)$ was expressed as a percentage according to the following equation.

$$
\operatorname{VEp}(\%)=\frac{P R U-P R V}{P R U} \times 100=\left(1-\frac{P R V}{P R U}\right) \times 100
$$

Progression rate in the unvaccinated patients (PRU) and progression rate in the vaccinated patients (PRV) were defined as the disease progression rates in non-vaccinated and vaccinated individuals, respectively. The $\mathrm{VE}_{\mathrm{p}}$ was derived as one minus the ratio of PRV to PRU, and the ratio of the progression rate was expressed as the odds ratio between the vaccination and unvaccination groups. The odds ratio and $95 \%$ confidence interval (CI) of the $\mathrm{VE}_{\mathrm{p}}$ were calculated by Fisher's exact test. Comparisons of baseline variables between the non-vaccination and valid vaccination groups (the invalid vaccination group was excluded from the analysis due to the small number of subjects) were performed using Fisher's exact tests for categorical value and Welch's $t$ tests for continuous values; $P<0.05$ was considered statistically significant. All statistical analyses were performed with R software, version 3.4.1 (R Foundation for Statistical Computing, Vienna, Austria). 


\section{Results}

A total of 192 HFRS subjects were enrolled in the program based on the study protocol. The reasons for exclusion included no available vaccination record $(n=12)$, absence of clinical data for severity estimation $(n=12)$, false-positive rapid immunochromatography assay findings due to prior vaccination $(n=24)$, and no evidence of renal involvement $(n=11)$. Among the 133 subjects, 23 had received at least one vaccination. According to the definition of valid vaccination, 18 patients were included in the valid vaccination group, while the other five patients were included in the invalid group due to the time elapsed since the last vaccination (Fig. 1).

Clinical parameters and event rates were compared within the non-vaccination $(n=110)$ and valid vaccination $(n=18)$ groups. The invalid vaccination group $(n=5)$ was excluded from analysis due to insufficient numbers. All patients were male with a mean age of 21.6 years (interquartile range, 20 to 22 years). No significant differences in age, baseline body weight, body mass index, and year at time of occurrence were observed. The patients' military rank at admission was significantly lower in the non-vaccination group compared to the vaccination group (Table 1).

The maximum weight gains from baseline were $8.0 \%$ and $8.2 \%$ in the non-vaccination and valid vaccination group ( $P=0.613)$, respectively. Clinical events such as pulmonary edema or intensive care unit (ICU) admission, mechanical ventilator use, renal replacement therapy, and inotropic agent administration did not differ significantly between the two groups. The clinical outcome expressed as KDIGO-AKI stage did not differ statistically between the two groups $(P=0.285)$. The proportion of patients with stage 3 AKI was $54.5 \%$ in the non-vaccination group and $33.3 \%$ in the vaccination group $(P=0.128)$. The duration of ICU stay among patients who received intensive care was 5.6 days in the non-vaccination group and 5.2 days in the valid vaccination group $(P=0.626)$. The mean length of hospitalization was 16.7 days in the non-vaccination group and 14.8 days in the vaccination group $(P=0.189)$ (Table 2$)$.

The $\mathrm{VE}_{\mathrm{p}}$ of the $\mathrm{iHV}$ was $58.1 \%$ as defined by stage $3 \mathrm{AKI}$ (95\% CI, $-31.3 \%$ to $88.0 \%$ ) and $57.3 \%$ as defined by di-

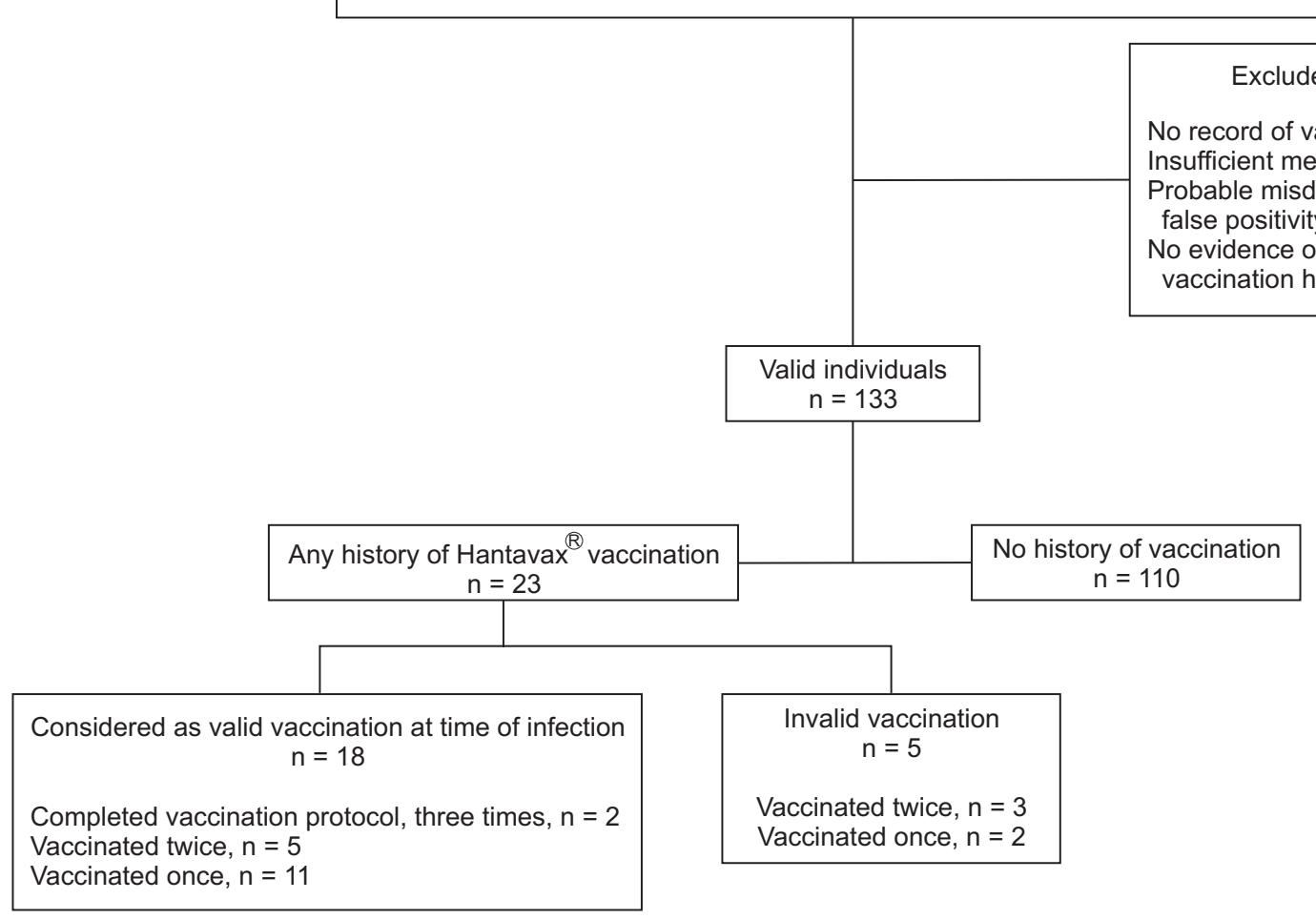

Figure 1. Flow diagram of patient disposition. Hantavax ${ }^{\circledR}$; Korea Green Cross, Seoul, Korea. 
alysis (95\% CI, $-102.1 \%$ to $95.5 \%)$. Neither $\mathrm{VE}_{\mathrm{p}}$ definition value showed statistical significance (Table 3 ).

While the peak serum aminotransferase level was significantly higher in the non-vaccination group (95\% CI, 13.0 to 118.7 ), the platelet and white blood cell counts, serum uric acid and creatinine levels, and urine protein/ creatinine ratio did not differ significantly between the groups (Table 4).

Table 2. Clinical outcomes of hemorrhagic fever with renal syndrome patients during hospitalization

\begin{tabular}{|c|c|c|c|c|}
\hline $\begin{array}{c}\text { Clinical } \\
\text { outcomes }\end{array}$ & $\begin{array}{l}\text { Non-vaccination } \\
\text { group }(n=110)\end{array}$ & $\begin{array}{l}\text { Valid vaccination } \\
\text { group }(n=18)\end{array}$ & $P$ value & $\begin{array}{l}\text { Invalid vaccination } \\
\text { group }(n=5)\end{array}$ \\
\hline Nadir daily urine output (mL) & $1,386(750-1,925)$ & $1,603(600-2,400)$ & 0.495 & \\
\hline Maximum gained weight from baseline (\%) & $8.0(4.5-11.2)$ & $8.6(5.8-11.2)$ & 0.613 & \\
\hline KDIGO-AKI stage & & & 0.285 & \\
\hline Stage 0 & $7(6.4)$ & $1(5.6)$ & & $1(20.0)$ \\
\hline Stage 1 & $17(15.5)$ & $5(27.8)$ & & 0 \\
\hline Stage 2 & $26(23.6)$ & $6(33.3)$ & & 0 \\
\hline Stage 3 & $60(54.5)$ & $6(33.3)$ & & $4(80.0)$ \\
\hline \multicolumn{5}{|l|}{ Clinical events } \\
\hline Pulmonary edema & $39(35.5)$ & $8(44.4)$ & 0.599 & 0 \\
\hline Mechanical ventilator therapy & $3(2.7)$ & 0 & 1 & 0 \\
\hline Hypotension & $16(14.5)$ & $4(22.2)$ & 0.482 & 0 \\
\hline Inotropic agents application & $12(10.9)$ & $2(11.1)$ & 1 & 0 \\
\hline Renal replacement therapy & $25(22.7)$ & $2(11.1)$ & 0.359 & 0 \\
\hline Mortality & $2(1.8)$ & 0 & 1 & 0 \\
\hline ICU admission events & $72(65.5)$ & $11(61.1)$ & 0.792 & $4(80.0)$ \\
\hline Duration of ICU care (d) & $5.6(4.0-6.5)$ & $5.2(4.0-7.0)$ & 0.626 & $9.8(6.8-14)$ \\
\hline Duration of total hospitalized care (d) & $16.7(12.0-21.0)$ & $14.8(10.3-17.8)$ & 0.189 & $23.2(19.0-32.0)$ \\
\hline
\end{tabular}

Data are presented as mean (interquartile range) or number (\%).

AKI, acute kidney injury; ICU, intensive care unit; KDIGO, Kidney Disease: Improving Global Outcomes.

Table 3. Effectiveness of the inactivated Hantaan virus vaccine on the disease progression

\begin{tabular}{lccc}
\hline & No vaccination group $(n=110)$ & Vaccination group $(n=18)$ & $V_{p}$ \\
\hline HFRS with KDIGO-AKI stage 3 & $60(54.5)$ & $6(33.3)$ & $58.1 \%(-31.3 \%$ to 88.0\%) \\
HFRS with renal replacement therapy & $25(22.7)$ & $2(11.1)$ & $57.3 \%(-102.1 \%$ to $95.5 \%)$ \\
\hline
\end{tabular}

Data are presented as number (\%) or odds ratio (95\% confidence interval).

AKI, acute kidney injury; HFRS, hemorrhagic fever with renal syndrome; KDIGO, Kidney Disease: Improving Global Outcomes; VE, vaccination effectiveness on progression.

Table 4. Laboratory parameters in hemorrhagic fever with renal syndrome patients

\begin{tabular}{lccc}
\hline \multicolumn{1}{c}{ Parameter } & No vaccination group $(\mathrm{n}=110)$ & Vaccination group $(\mathrm{n}=18)$ & $P$ value \\
\hline Peak serum creatinine $(\mathrm{mg} / \mathrm{dL})$ & $3.85(1.89-4.98)$ & $3.19(1.71-4.50)$ & 0.283 \\
Nadir platelets counts $(/ \mu \mathrm{L})$ & $38,455(20,250-51,750)$ & $45,000(28,000-56,000)$ & 0.333 \\
Peak WBC counts $(/ \mu \mathrm{L})$ & $19,326(11,942-25,098)$ & $17,879(13,440-21,220)$ & 0.498 \\
Peak serum aminotransferase level $(\mathrm{IU} / \mathrm{L})$ & $199(94-222)$ & $133(63-184)$ & 0.015 \\
Peak serum uric acid level $(\mathrm{mg} / \mathrm{dL})$ & $10.3(7.6-12.4)$ & $11.5(8.5-12.6)$ & 0.544 \\
Peak urine protein/creatinine ratio $(\mathrm{g} / \mathrm{g})$ & $4.37(2.02-5.87)$ & $5.30(3.45-6.91)$ & 0.279 \\
\hline
\end{tabular}

Data are presented as mean (interquartile range).

WBC, white blood cell. 


\section{Discussion}

The effectiveness of the iHV on disease progression in HFRS patients was investigated. The $\mathrm{VE}_{\mathrm{p}}$ values were $58.1 \%$ for stage $3 \mathrm{AKI}$ and $57.3 \%$ for patients with AKI who received renal replacement therapy. The overall prevalence of severe cases was $52.6 \%$ (70 of 133 HFRS patients) when defined as stage $3 \mathrm{AKI}$ and $20.3 \%$ when defined as receiving renal replacement therapy when defined as stage 3 AKI (27 of 133 patients). Only two cases of mortality occurred during the study period, and neither patient was vaccinated. Therefore, the iHV may have moderated HFRS disease severity in the military population.

Another studies have reported the efficacy of the iHV on the severity of HFRS [12]. Two studies included a control group within an ROK Army battalion in an HFRS endemic area $[13,14]$. The vaccine effectiveness against disease onset was calculated by comparison of HFRS and nonHFRS patients in the same hospital and showed a statistically significant effectiveness of the iHV to reduce disease prevalence by approximately $60 \%$ [14]. In contrast, our study assessed the vaccine effectiveness based on HFRS disease progression, assessing HFRS severity among iHV vaccination and non-vaccination groups.

This study is also characterized by vaccination status of study subjects. Only two of 23 vaccinated patients completed the three dose protocol of the iHV. Several studies evaluating iHV immunogenicity have consistently reported high positive seroconversion rate confirmed by immunofluorescent antibody assay after vaccination. The reported seroconversion rate at one month after the first dose ranged from $50 \%$ to $80 \%$, which increases to $80-100 \%$ after the second dose. The seroconversion rate then dropped at 12 months in approximately $30 \%$ to $40 \%$ of subjects. An iHV booster dose at 13 months successfully raises the seroconversion rate by $90 \%$ to $100 \%$ [7,8,11]. In this study, 13 of 23 patients had received only one iHV dose at the time of disease occurrence. Another HFRS study in the ROK Army showed vaccination status in HFRS patients as $37 \%$ for one dose, $35 \%$ for two doses, and $28 \%$ for three doses [13]. While the complexity of vaccination status among patients was suited for practical application, further study of dose-related efficacy of $\mathrm{iHV}$ is required.

This study has several limitations. Due to its retrospective and observational study design, mild cases were in- cluded in the preliminary data of the vaccination group. However, preliminary registration and medical history record review identified that more than half of the vaccinated patients were misdiagnosed with HFRS due to false-positive rapid immunochromatography assay. We confirmed the status of the valid-vaccinated HFRS cases by excluding all uncertain cases by clinical misdiagnosis and false-positive serological test findings. In comparison, fewer HFRS unvaccinated patients were excluded; less than one-tenth of the unvaccinated subjects did not meet the clinical criteria of HFRS. The selective bias in these groups may have affected the true vaccination effect on disease progression. If the less severe cases were more often excluded in the vaccination group compared to the non-vaccination group, the $\mathrm{VE}_{\mathrm{p}}$ may have been underestimated. Second, the value of vaccination effectiveness on disease progression failed to show statistical significance. This result was partially attributed to insufficient numbers of validly vaccinated subjects. Furthermore, the unequal numbers of subjects between the two groups resulted in nonparametric analysis, lowering the statistical test values. Lastly, differences in the study population characteristics prevented generalization of the results to the entire population. All of the patients in this study were men, the vast majority of which were in their twenties. Since sex differences in the incidence and mortality of HFRS have been reported, further research is needed to determine the effectiveness of the iHV program in the general population $[15,16]$.

In conclusion, this study described the clinical presentations and disease courses of patients with HFRS in South Korean military areas and compared two groups according to vaccination status. Vaccination appeared to reduce HFRS progression. The prevalence of severe renal disease, defined as stage $3 \mathrm{AKI}$ or receiving renal replacement therapy, among iHV vaccinated patients was approximately half that of unvaccinated patients. This result implies a positive preventive effect of the iHV against severe HFRS outcomes. The effectiveness of the iHV was suspected to affect not only HFRS incidence, but also HFRS progression. However, because the test value of the analysis in this study did not reach statistical significance, further studies with larger numbers of subjects are required. 


\section{Conflicts of interest}

All authors have no conflicts of interest to declare.

\section{Acknowledgments}

This work was supported by the Korean Military Medical Research Project funded by the ROK Ministry of National Defense (ROK-MND-2017-KMMRP-029).

\section{References}

[1] Hart CA, Bennett M. Hantavirus infections: epidemiology and pathogenesis. Microbes Infect 1:1229-1237, 1999

[2] Lee HW, Lee PW, Johnson KM. Isolation of the etiologic agent of Korean hemorrhagic fever. J Infect Dis 137:298308, 1978

[3] Bi Z, Formenty PB, Roth CE. Hantavirus infection: a review and global update. J Infect Dev Ctries 2:3-23, 2008

[4] Muranyi W, Bahr U, Zeier M, van der Woude FJ. Hantavirus infection. J Am Soc Nephrol 16:3669-3679, 2005

[5] Baek LJ, Kariwa H, Lokugamage K, et al. Soochong virus: an antigenically and genetically distinct hantavirus isolated from Apodemus peninsulae in Korea. J Med Virol 78:290297, 2006

[6] Krüger DH, Schönrich G, Klempa B. Human pathogenic hantaviruses and prevention of infection. Hum Vaccin 7: 685-693, 2011

[7] Cho HW, Howard CR. Antibody responses in humans to an inactivated hantavirus vaccine (hantavax). Vaccine 17: 2569-2575, 1999

[8] Cho HW, Howard CR, Lee HW. Review of an inactivated vaccine against hantaviruses. Intervirology 45:328-333, 2002

[9] Song JW, Moon SS, Gu SH, et al. Hemorrhagic fever with renal syndrome in 4 US soldiers, South Korea, 2005. Emerg Infect Dis 15:1833-1836, 2009

[10] Heo JY, Choe KW, Yoon CG, Jeong HW, Kim WJ, Cheong HJ. Vaccination policy in Korean armed forces: current status and future challenge. J Korean Med Sci 30:353-359, 2015

[11] Song JY, Woo HJ, Cheong HJ, Noh JY, Baek LJ, Kim WJ. Longterm immunogenicity and safety of inactivated Hantaan virus vaccine (HantavaxTM) in healthy adults. Vaccine 34: 1289-1295, 2016

[12] Oh MD, Lee JK. Milestones in history of adult vaccination in Korea. Clin Exp Vaccine Res 1:9-17, 2012.

[13] Park K, Kim CS, Moon KT. Protective effectiveness of hantavirus vaccine. Emerg Infect Dis 10:2218-2220, 2004

[14] Jung J, Ko SJ, Oh HS, et al. Protective effectiveness of inactivated hantavirus vaccine against hemorrhagic fever with renal syndrome. J Infect Dis 217:1417-1420, 2018

[15] Klein SL, Marks MA, Li W, et al. Sex differences in the incidence and case fatality rates from hemorrhagic fever with renal syndrome in China, 2004-2008. Clin Infect Dis 52: 1414-1421, 2011

[16] Lee SH, Chung BH, Lee WC, Choi IS. Epidemiology of hemorrhagic fever with renal syndrome in Korea, 2001-2010. J Korean Med Sci 28:1552-1554, 2013 